\title{
Symptome von Erkrankungen der Augenoberfläche bei Bauarbeitern: eine vergleichende Studie mit Büroangestellten
}

\author{
Sergio Hernandez-Llamas ${ }^{a}$ Ana Karen Paz-Ramos ${ }^{a}$ Patricio Marcos-Gonzalez ${ }^{a}$ \\ Francisco Amparo ${ }^{a, b}$ Manuel Garza-Leon ${ }^{a}$ \\ ${ }^{a}$ Department of Clinical Sciences, Division of Health Sciences, University of Monterrey, San Pedro Garza García, Mexiko; ${ }^{b}$ Cornea Service, \\ Mass Eye, and Ear, Department of Ophthalmology, Harvard Medical School, Boston, MA, USA
}

\author{
Schlüsselwörter \\ Symptome des trockenen Auges · OSDI · Berufsrisiko · Büro · \\ Bauarbeiter
}

\section{Zusammenfassung}

Hintergrund: Mit der vorliegenden Studie sollte die Prävalenz von Symptomen des trockenen Auges bei Bauarbeitern im Vergleich zu Büroangestellten mithilfe des OSDI-Fragebogens untersucht werden.

Methoden: Es wurde eine Querschnitts-Beobachtungsstudie durchgeführt, bei der die Symptome des trockenen Auges und die damit verbundenen Risikofaktoren anhand des OSDI-Fragebogens bewertet wurden. Die Berechnung der Stichprobengröße mit einer Power von $80 \%$ und einem Konfidenzniveau von 95\% ergab, dass 298 Teilnehmer in die Studie aufzunehmen waren.

Ergebnisse: Wir untersuchten 304 Probanden (149 Bauarbeiter und 155 Büroangestellte). Mehr als die Hälfte (55\%) der Teilnehmer zeigten Symptome des trockenen Auges (OSDI > 12). Der durchschnittliche OSDI-Score betrug 21,30 $\pm 22,20$ Punkte und fiel in der
Gruppe der Bauarbeiter $(12,45 \pm 17,50)$ niedriger aus als bei den Büroangestellten $(28,51 \pm 22,99)(p<0,001)$. Bei Betrachtung der Teilnehmer mit mittelschweren und schweren Symptomen (23 bis 100 Punkte im OSDI) hatten Büroangestellte 4,15mal häufiger Symptome des trockenen Auges als Bauarbeiter (OR: 4,15, 95\%-Kl: 2,52, $6,85)$. Frauen wiesen statistisch höhere OSDI-Scores auf als Männer $(32,47 \pm 23,72$ vs. $14.87 \pm 18,48)$

Schlussfolgerungen: Bei Bauarbeitern ist das Risiko für Symptome des trockenen Auges viermal niedriger als bei Menschen, die in einer durchschnittlichen Büroumgebung arbeiten. Dies zeigt die schädlichen Effekte der Büroumgebung auf die Augenoberfläche, die ein erhebliches Risiko für die Entwicklung oder Verschlimmerung von Symptomen des trockenen Auges darstellen.

(c) 2020 The Author(s)

\section{Hintergrund}

Symptome von trockenen und gereizten Augen treten bei vielen Berufsgruppen häufig auf und können starke Auswirkungen auf die Produktivität am Arbeitsplatz haben [1]. Der neue TFOS DEWS II-Konsens hebt die Bedeutung der Symptomatik für das diagnostische Vorgehen beim trockenen Auge hervor, da sie die Grundlage für die Klassifizierung eines Patienten mit dieser Krankheit bildet [2]. Fragebögen sind das weltweit am häufigsten eingesetzte Instrument für die Diagnose und Verlaufskon- trolle des Syndroms des trockenen Auges, da sie einfach in der Anwendung sind und die Möglichkeit bieten, große Populationen zu geringen Kosten zu untersuchen sowie die Auswirkungen der Erkrankung auf die Lebensqualität der Patienten zu bewerten [2]. Es gibt mehr als 15 verschiedene Fragebögen, die bei der Diagnose von Symptomen des trockenen Auges (dry eye symptoms, DES) zur Anwendung kommen und von denen drei die Auswirkungen der Erkrankung auf die Lebensqualität erfassen (Impact of Dry Eye on Everyday Life [3], Dry Eye-Related Quality-of-Life Score [4] und Ocular Surface Disease Index 
(OSDI) [5]). Gemäß den TFOS DEWS II-Konsensempfehlungen sollte der OSDI verwendet werden, da dieser auf diesem Gebiet sehr verbreitet ist [2].

Der OSDI-Fragebogen [5] ist in epidemiologischen DES-Studien weit verbreitet [2]. Er umfasst 12 Fragen zur Bewertung der Symptome und ihre Auswirkungen auf die Lebensqualität der Patienten in drei Domänen: okuläre Symptome, sehkraftbezogene Funktionen und umweltbedingte Auslöser. Jede Frage wird anhand einer Skala von 0 bis 4 bewertet, und das Endergebnis des OSDI-Scores kann zwischen 0 und 100 betragen. Entsprechend dem OSDI-Score können die Patienten folgenden Kategorien zugeordnet werden: «normale» Augenoberfläche (0 bis 12 Punkte), «leichte» Symptome des trockenen Auges (13 bis 22 Punkte), «mittelschwere» Symptome (23 bis 32 Punkte) oder «schwere» Symptome des trockenen Auges (33 bis 100 Punkte) [6].

Die Anzahl der Studien, die die Auswirkungen der Arbeitsumgebung auf die Prävalenz von Symptomen des trockenen Auges untersucht haben, ist begrenzt, und in den meisten dieser Studien lag der Schwerpunkt auf herkömmlichen Innenraum-Arbeitsplätzen. In der OFFICAIR-Studie wurden Mitarbeiter in 167 Bürogebäuden in 8 europäischen Ländern untersucht und festgestellt, dass 34\% der befragten Teilnehmer Symptome des trockenen Auges zeigten [7]. Nach Untersuchungen von Uchino et al. in Japan wiesen 26\% bis 48\% der Angestellten in 4 unterschiedlichen Büroräumen schwere Symptome des trockenen Auges auf [8]. Neben den Untersuchungen zur Prävalenz haben andere Studien den Versuch unternommen, die mit Symptomen des trockenen Auges verbundenen Risikofaktoren bei Büroangestellten zu ermitteln [9, 10]. Die Ursache von Beschwerden und anderen okulären Symptomen in Büroumgebungen ist multifaktoriell und steht möglicherweise mit umweltbedingten, berufsbezogenen und individuellen Faktoren in Zusammenhang [9]. Zu den individuellen Faktoren gehören unter anderem Geschlecht, Rauchgewohnheiten, psychosoziale Belastung und ein sitzender Lebensstil. Mögliche Umweltfaktoren sind Luftverschmutzung, Feinstaub, Temperatur, niedrige Luftfeuchtigkeit und Exposition gegenüber Videomonitoren [8-12]. Eine Analyse des Health and Nutrition Examination Survey in Südkorea in Hinblick auf die verschiedenen Berufe zeigte, dass das DES-Risiko bei Fachkräften und Technikern im Vergleich zu Landarbeitern erhöht ist [13]. Gupta et al. berichteten über eine Prävalenz des DES von 33\% unter Gerbereiarbeitern In Indien [14]. Über das Auftreten von DES bei Bauarbeitern liegen jedoch nur wenige Berichte vor.

Wir vermuteten, dass die anhaltende Exposition gegenüber Baumaterialien, Staub oder Schutt die Häufigkeit von DES erhöht. Mit der vorliegenden Studie sollte die Häufigkeit von Symptomen des trockenen Auges bei Bauarbeitern im Vergleich zu Büroangestellten mithilfe einer validierten spanischsprachigen Version des OSDI-Fragebogens untersucht werden [15].

\section{Hypothese}

Die Häufigkeit von Symptomen des trockenen Auges ist bei Bauarbeitern höher als die durchweg für Büroangestellte berichtete Häufigkeit, was vor allem an der anhaltenden Exposition gegen- über Staub, Schutt, reizstoffhaltigen Baumaterialien und anderen Umweltfaktoren liegt.

\section{Methoden}

Bei der vorliegenden Studie handelte sich um eine deskriptive Querschnitts-Beobachtungsstudie, bei der das Vorliegen von Symptomen des trockenen Auges (Ocular Surface Disease Index) und der damit verbundenen Risikofaktoren wie Arbeitszeiten, die täglich am Computer oder Bildschirm verbrachte Zeit, das Tragen von Kontaktlinsen, Schweißarbeiten und das Tragen von Schutzbrillen bei Bauarbeitern oder die Büroarbeitsumgebung (offene Bürobereiche vs. geschlossene Büros) sowie okuläre oder systemische Erkrankungen (definiert als eine frühere Diagnose oder Behandlung gemäß ärztlichen Angaben) anhand eines standardisierten Fragebogens mit insgesamt 24 Fragen beurteilt wurde. In beiden Gruppen mussten die Probanden mindestens 18 Jahre alt und bereit sein, an der Studie teilzunehmen und sie mussten seit mindestens 6 Monaten auf ihrer aktuellen Arbeitsstelle gearbeitet haben. Die Studie wurde zwischen Oktober und Dezember 2017 durchgeführt. Sie wurde durch die Behörden und die Ethikkommission der Universität von Monterrey (UDEM) genehmigt und entsprach den Prinzipien der Deklaration von Helsinki. Nach Aufklärung über die Studie wurde von allen Teilnehmern eine mündliche Einwilligungserklärung eingeholt. Diese Art der Einwilligung wurde von der Ethikkommission der Universität von Monterrey genehmigt, weil viele Studienteilnehmer (insbesondere die Bauarbeiter) nur eingeschränkte Lese- und Schreibfähigkeiten besaßen und weil die Erteilung der informierten Einwilligung am Arbeitsplatz unter suboptimalen Bedingungen und während Arbeitszeit erfolgen musste. Dadurch wurde die Zeit, die für die Einholung der Einwilligung nach Aufklärung und das Interview zur Verfügung stand, erheblich eingeschränkt. Es handelte sich um eine nicht-interventionelle, risikoarme Studie und es wurden keine vertraulichen persönlichen Daten von den Teilnehmern erhoben.

\section{Stichprobenumfang}

Die Zielpopulation waren die Angestellten im UDEM-Rektoratsbüro und Bauarbeiter, die auf einem neuen Campus-Modul arbeiteten. Die Baustelle befindet sich auf dem Universitätscampus in einem städtischen Bereich neben stark befahrenen Straßen.

\section{Stichprobenverfahren}

Es wurde ein randomisiertes Stichprobenverfahren verwendet, d.h. es bestand für jede zur Grundpopulation gehörende Person die gleiche Wahrscheinlichkeit, für die Stichprobe ausgewählt zu werden. Bauarbeiter, die am Aushub der Fundamente arbeiteten, wurden zur Teilnahme während der Arbeitszeit (zwischen 8 bis 18 Uhr) eingeladen und dort von einem Prüfer besucht. Büroangestellte wurden während der Arbeitszeit von einem Prüfer an ihrem Arbeitsplatz besucht. Alle Probanden, die die Auswahlkriterien erfüllten und zur Teilnahme bereit waren, wurden in die Stichprobe eingeschlossen. Sie wurden über die Studie aufgeklärt 
Tab. 1. Demographische Merkmale und Risikofaktoren

\begin{tabular}{|c|c|c|c|c|}
\hline Variable & $\begin{array}{l}\text { Gesamt } \\
n=304(100 \%)\end{array}$ & $\begin{array}{l}\text { Bauarbeiter } \\
n=149(49 \%)\end{array}$ & $\begin{array}{l}\text { Büroangestellte } \\
n=155(51 \%)\end{array}$ & $p(95 \%-\mathrm{KI})$ \\
\hline Alter (SD), Jahre & $34,04 \pm 10,63$ & $35,12 \pm 11,40$ & $33,03 \pm 9,77$ & $0,08^{\mathrm{a}}(-0,32,4,50)$ \\
\hline Geschlecht M/F (\%) & $193 / 111(63,5 / 36,5)$ & $147 / 2(98,7 / 1,3)$ & $46 / 109(29,7 / 70,3)$ & $<0,0001^{b}$ \\
\hline Raucher (\%) & $94(30,9)$ & $66(44,3)$ & $28(18,1)$ & $<0,0001^{b}$ \\
\hline Kontaktlinsenträger (\%) & $25(8,2)$ & $2(1,3)$ & $23(14,8)$ & $0,0001^{b}$ \\
\hline Am Computer verbrachte Stunden pro Tag & $4,39 \pm 4,24$ & $0,40 \pm 1,41$ & $8,19 \pm 1,86$ & $<0,0001^{c}$ \\
\hline Arbeitsstunden pro Tag & $9,38 \pm 1,90$ & $9,63 \pm 2,03$ & $8,56 \pm 1,02$ & $0,0001^{\mathrm{a}}(0,48,1,69)$ \\
\hline Augenerkrankung & $48(15,8)$ & $15(10,1)$ & $33(21,3)$ & $0,007^{b}$ \\
\hline Systemerkrankung & $21(6,9)$ & $9(6)$ & $12(7,7)$ & $0,55^{b}$ \\
\hline
\end{tabular}

a Student- $t$-Test, ${ }^{\mathrm{b}} \mathrm{X}^{2}$-Test, ${ }^{\mathrm{c}}$ Mann-Whitney-Test.

Tab. 2. Verteilung der Symptome einer Augenoberflächenerkrankung

\begin{tabular}{|c|c|c|c|c|c|}
\hline Variable & Alter & $\begin{array}{l}\text { Gesamt } \\
n=304(100 \%)\end{array}$ & $\begin{array}{l}\text { Bauarbeiter } \\
n=149(49 \%)\end{array}$ & $\begin{array}{l}\text { Büroangestellte } \\
n=155(51 \%)\end{array}$ & $p(95 \%-K I)$ \\
\hline OSDI & & $21,30 \pm 22,20$ & $12,45 \pm 17,50$ & $28,51 \pm 22,99$ & $0,001^{\mathrm{a}}(-21,99,-12,75)$ \\
\hline Symptomfrei (\%) & $31,55 \pm 9,02$ & $139(45,9)$ & $96(64,4)$ & $43(27,7)$ & $<0,001^{b}$ \\
\hline Leichte Symptome (\%) & $35,17 \pm 11,78$ & $48(15,8)$ & $20(13,4)$ & $28(18,1)$ & $<0,001^{b}$ \\
\hline Mittelschwere Symptome (\%) & $41,60 \pm 10,28$ & $35(11,5)$ & $14(9,4)$ & $21(13,5)$ & $<0,001^{b}$ \\
\hline Schwere Symptome (\%) & $34,33 \pm 11,06$ & $82(26,9)$ & $19(12,8)$ & $63(40,6)$ & $<0,001^{b}$ \\
\hline
\end{tabular}

a Student- $t$-Test, ${ }^{b} X^{2}$-Test.

und unterzeichneten eine Einverständniserklärung, bevor die Befragung durchgeführt wurde. Büroangestellte und Bauarbeiter beantworteten die OSDI-Fragebögen durch Selbstauskunft unter Aufsicht eines Prüfers, der in allen Fällen derselbe war und alle unbekannten Begriffe erklärte. Die Prüfer griffen bei der Beantwortung der Fragen durch den Probanden nicht ein. Nach Beantwortung des OSDI-Fragebogens wurden die jeweiligen demographischen Daten und die Risikofaktoren erfasst.

\section{Berechnung des Stichprobenumfangs}

Die Berechnung des Stichprobenumfangs erfolgte mithilfe einer Formel zum Vergleich unabhängiger Proportionen entsprechend den von der OFFICAIR-Studie [7] beschriebenen Proportionen mit einer erwarteten Ereignisrate von 34\% für Büroangestellte und $50 \%$ für Bauarbeiter und mit einer Power von $80 \%$ und sowie einem Konfidenzniveau von 95\%. Die berechneten Ergebnisse sprachen für den Einschluss von 298 Teilnehmern, aufgeteilt in 2 Gruppen zu je 149 Probanden.

\section{Statistische Auswertung}

Wir ermittelten in beiden Gruppen die Prävalenz von Symptomen des trockenen Auges sowie den Zusammenhang zwischen den untersuchten Risikofaktoren und den OSDI-Scores unter Berücksichtigung der folgenden Faktoren: 1) Geschlecht, 2) Rauchgewohnheiten, 3) Kontaktlinsenverwendung, 4) am Computer verbrachte Stunden, 5) Augenkrankheiten und 6) systemische Erkrankungen.
Die Odds Ratios (OR) und ihre 95\%-Konfidenzintervalle (95\%KIs) wurden berechnet, um den Zusammenhang zwischen DES und Risikofaktoren zu bestimmen. Bei der OR-Analyse galten Probanden, die mittelschwere und schwere Symptome (23 bis 100 Punkte im OSDI) hatten, als Personen mit eindeutigem Vorliegen von Symptomen einer Augenoberflächenerkrankung (ocular surface disease, OSD) [6].

Die Prävalenz von Symptomen des trockenen Auges wurde berechnet und ihr Zusammenhang mit den Faktoren Geschlecht, Rauchgewohnheiten, Anzahl der Arbeitsstunden, Kontaktlinsenverwendung, am Computer verbrachte Stunden, Anwendung von Augentropfen und systemischen und okulären Erkrankungen erfolgte mittels bivariater und multivariater logistischer Regressionsanalyse, bei der Interaktionen und mögliche Einflüsse zwischen den untersuchten Variablen ausgeglichen werden. Für die Analyse normalverteilter Daten wurden ein X2-Test, ein Studentt-Test und eine einseitige ANOVA mit Post-hoc-Scheffé-Test verwendet. Bei nicht normalverteilen Daten kam der Mann-Whitney-Test zur Anwendung. Das gewählte statistische Signifikanzniveau lag bei $p<0,05$. Die Analyse der Daten erfolgte mit dem Statistikpaket SPSS V.21 (IBM Corp, Armonk, NY, USA).

\section{Ergebnisse}

Insgesamt wurden 304 Personen untersucht, von denen 51\% im Büro und 49\% auf dem Bau arbeiteten. Das Durchschnittsalter betrug 34,04 \pm 10,63 Jahre und es bestanden keine signifikanten 
Tab. 3. Merkmale der Büroangestellten

\begin{tabular}{|c|c|c|c|c|}
\hline Variable & $\begin{array}{l}\text { Gesamt } \\
n=155(100 \%)\end{array}$ & $\begin{array}{l}\text { Geschlossenes Büro } \\
n=95(61,3 \%)\end{array}$ & $\begin{array}{l}\text { Offener Bürobereich } \\
n=60(38,7 \%)\end{array}$ & $p(95 \%-K I)$ \\
\hline Alter (SD), Jahre & $33,03 \pm 9,77$ & $32,27 \pm 9,13$ & $34,22 \pm 10,66$ & $0,22^{\mathrm{a}}(-1,2,5,1)$ \\
\hline Geschlecht M/F (\%) & $46 / 109(29,7 / 70,3)$ & $31 / 64(32,6 / 67,4)$ & $15 / 45(25 / 75)$ & $0,31^{b}$ \\
\hline Raucher (\%) & $28(18,1)$ & $23(24,2)$ & $5(8,3)$ & $0,1^{b}$ \\
\hline Kontaktlinsenträger (\%) & $23(14,8)$ & $15(15,8)$ & $8(13,3)$ & $0,67^{b}$ \\
\hline Am Computer verbrachte Stunden pro Tag & $8,19 \pm 1,86$ & $8,24 \pm 1,79$ & $8,20 \pm 1,81$ & $0,87^{c}$ \\
\hline Arbeitsstunden pro Tag & $8,56 \pm 1,02$ & $8,47 \pm 1,60$ & $8,53 \pm 1,26$ & $0,80^{\mathrm{a}}(-0,4,0,5)$ \\
\hline Augenerkrankung & $33(21,3)$ & $15(15,8)$ & $18(30)$ & $0,03^{b}$ \\
\hline Systemerkrankung & $12(7,7)$ & $7(7,4)$ & $5(8,3)$ & $0,82^{b}$ \\
\hline
\end{tabular}

Altersunterschiede zwischen den beiden Gruppen. Von den Probanden waren 193 Männer (63,5\%) und 111 Frauen $(p<$ 0,0001). Die demographischen Merkmale sind in Tabelle 1 aufgeführt.

Der mittlere OSDI-Score betrug 21,30 $\pm 22,20$ und die Gruppe der Bauarbeiter wies signifikant niedrigere mittlere DES-Symptome auf als die Gruppe der Büroangestellten (OSDI-Score 12,45 \pm $17,50$ vs. $28,51 \pm 22,99)(p=<0,001)$ (Tabelle 2$)$. Der Unterschied in den OSDI-Scores zwischen Bauarbeitern und Büroangestellten blieb auch dann statistisch signifikant, wenn nur die männlichen Teilnehmer verglichen wurden $(12,60 \pm 17,44$ vs. $22,12 \pm 20,01)$ ( $p=0,02,95 \%$-KI -15,53, -3,48). Unter allen Arbeitern und Angestellten hatten Frauen statistisch signifikant höhere DES-Symptome als Männer (OSDI 32,47 $\pm 23,72$ vs. 14,87 $\pm 18,48 ; p<0,001$; 95\%-KI -12,43, 22,75).

Mehr als die Hälfte der Teilnehmer (55\%) hatten leichte Symptome des trockenen Auges (OSDI-Score 12 bis 23). Teilnehmer mit mittelschweren DES-Symptomen waren älter als Teilnehmer ohne DES $(p<0,001)$, mit leichten DES-Symptomen $(p=0,04)$ oder schweren DES-Symptomen $(p<0,01)$. Die Gruppen und die allgemeine Verteilung sind in Tabelle 2 dargestellt.

Kontaktlinsenträger hatten schwerere DES-Symptome als NichtKontaktlinsenträger $(35,25 \pm 22,46$ vs. $20,05 \pm 21,78)$ und der Unterschied zwischen den beiden Gruppen war statistisch signifikant $(p<0,001,95 \%-K I-24,17,-6,23)$. Bei Rauchern fielen die Symptome des trockenen Auges geringer aus als bei Nichtrauchern (17,56 $\pm 19,93$ vs. $22,97 \pm 22,99 ; p=0,04$ [95\%-KI -0, 01, -10 , 80]); ein Unterschied, der bei separater Analyse der beiden Gruppen jedoch verschwand.

Achtzehn (18) Probanden wiesen eine Augenerkrankung auf, bei der es sich nicht um Fehlsichtigkeit handelte. Von diesen hatten 4 ein diagnostiziertes Trockenes Auge und verwendeten künstliche Tränen, 3 hatten ein diagnostiziertes Pterygium oder Pinguecula, 2 hatten die Diagnose «allergische Konjunktivitis», die keine Behandlung erforderte, 3 Teilnehmer hatten die Diagnose «Glaukom», das mit Prostaglandin-Analoga behandelt wurde, und 3 eine diagnostizierte Katarakt. Bei 19 Teilnehmern lag eine Systemerkrankung vor; von diesen hatten 7 Probanden Diabetes mellitus Typ 2, 5 hatten Allergien oder Asthma und 3 Probanden hatten eine Hypertonie. Eine Teilnehmerin nahm orale Kontrazeptiva ein.

Aufgrund der Art der Arbeit war die Zahl der männlichen Probanden in der Bauarbeitergruppe höher, und sie arbeiteten seltener am Computer. Außerdem gaben sie seltener an, Kontaktlinsen zu tragen und rauchten häufiger als Büroangestellte (Tabelle 1). Die durchschnittliche Arbeitszeit am Tag betrug 9,38 $\pm 1,90$ Stunden und war in der Gruppe der Bauarbeiter länger (9,63 \pm $2,03)$ als bei den Büroangestellten $(8,56 \pm 1,02)(p<0,001)$.

Die 95 Büroangestellten, die in einem geschlossenen Büro arbeiteten, hatten signifikant geringere DES-Symptome als Angestellte, die in offenen Bereichen (Bürowaben) arbeiteten (OSDI-Score $26,52 \pm 21,37$ vs. $35,02 \pm 24,72)(p=0,03,95 \%-K I-16,17,-0,82)$. Die demografischen Merkmale und Risikofaktoren sind in Tabelle 3 aufgeführt.

Büroangestellte hatten 4,15mal häufiger Symptome des trockenen Auges als Bauarbeiter (OR 4,15. 95\%-KI 2,52, 6,85), und dieses Ergebnis blieb auch dann bestehen, wenn nur die Männer verglichen wurden (OR 2,43, 95\%-KI 1,20, 4,91). Bei der separaten Auswertung zeigte sich, dass Frauen 3,82mal häufiger DES-Symptome hatten als Männer (OR 3,82, 95\%-KI 2,48, 5,91). Unter den Büroangestellten hatten Frauen häufiger DES-Symptome als Männer (OR 2,09, 95\%-KI 1,04, 4,23). Kontaktlinsenträger wiesen ein 4,67mal höheres DES-Risiko auf als diejenigen, die keine Kontaktlinsen trugen (95\%-KI 1,88, 11,57) (Abb. 1).

Abbildung 2 zeigt die multivariate DES-Risikoanalyse nach Adjustierung für Arbeitsgruppe, Geschlecht, Rauchgewohnheiten, Kontaktlinsenverwendung sowie okuläre und systemische Erkrankungen Die mittlere relative Luftfeuchtigkeit während der Studientage betrug im Bürogebäude 40,3 $\pm 1,37 \%$ (Spanne: 38 bis $43 \%$ ) und im Freien auf der Baustelle 59,75 $\pm 2,62 \%$ (Spanne: 54 bis 63\%) $(p<0,01)$.

\section{Diskussion}

In der vorliegenden Studie wurde der Einfluss von Umweltfaktoren auf das Auftreten von Symptomen des trockenen Auges bei Bauarbeitern im Vergleich zu Büroangestellten untersucht. Dabei stellten wir fest, dass Büroangestellte schwerere Symptome 


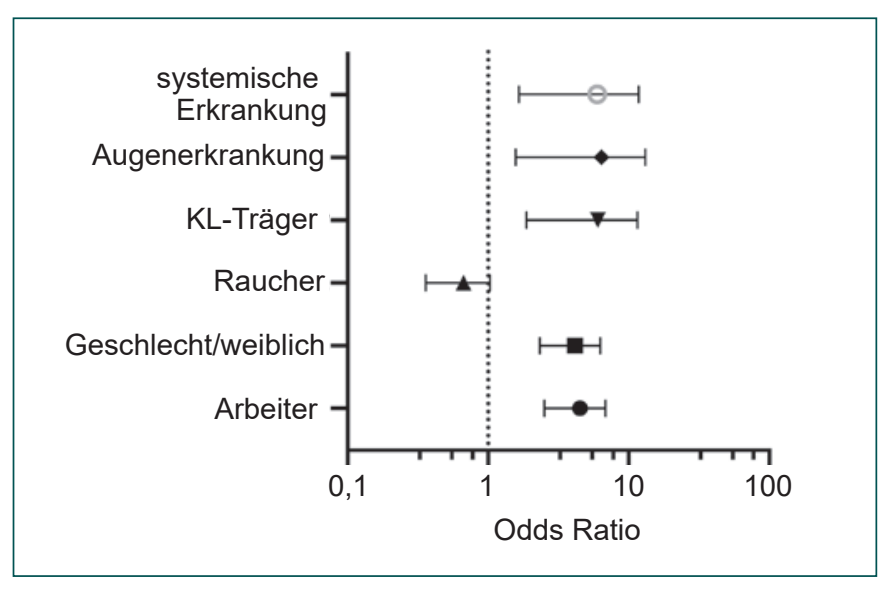

Abb. 1. Odds Ratio der univariaten DES-Risikoanalyse.

hatten als Bauarbeiter, und das DES-Risiko blieb auch nach Anpassung für alle untersuchten Variablen bestehen. Diese Ergebnisse können durch verschiedene Faktoren beeinflusst worden sein, möglicherweise jedoch vor allem durch die anhaltende Computerarbeit der Büroangestellten in deutlich trockenerer Umgebung, denn in unserer Studie war die Büroumgebung um $50 \%$ trockener als die Außenluft. Ein Bericht von Kawashima et al. stützt diese Ergebnisse. Sie zeigten, dass Symptome des trockenen Auges häufig von Personen berichtet werden, die Bildschirme benutzen [12]. Uchino et al. berichteten ebenfalls über ein erhöhtes DES-Risiko, wenn mehr als $4 \mathrm{~h}$ am Bildschirm verbracht werden [8]. Lee et al. untersuchten 6023 Probanden und zeigten, dass neben der Computernutzung auch andere Umweltfaktoren Einfluss auf die Entwicklung von Symptomen des trockenen Auges haben können. In ihrer Untersuchung hatten 16\% der Teilnehmer DES-Symptome, wobei das DES-Risiko bei Fachkräften, Managern, Führungskräften und leitenden Angestellte höher war als bei Fabrikarbeitern. Ferner berichteten sie, dass Menschen, die im Freien arbeiten, ein niedrigeres DES-Risiko aufweisen [13].

In der vorliegenden Studie fielen die OSDI-Scores bei Frauen höher aus als bei Männern, und diese Beobachtung steht im Einklang mit zahlreichen anderen Berichten [8, 13, 15-22]; infolgedessen könnten die Unterschiede zwischen den Gruppen durch Geschlechtsunterschiede beeinflusst worden sein, da der Anteil der Frauen unter den Büroangestellten höher war. Nach Bereinigung für das Geschlecht in den multivariaten Analysen waren jedoch keine statistisch signifikanten Unterschiede zwischen den Geschlechtern mehr nachweisbar.

Das Tragen von Kontaktlinsen wurde mit DES in Verbindung gebracht $[15,18,20,23,24]$, und in unserer Studie wiesen Kontaktlinsenträger einen höheren Score beim OSDI-Fragebogen auf als Nicht-Kontaktlinsenträger. Bei der multivariaten Analyse verringerte dieser Einfluss jedoch die OR, was wahrscheinlich darauf zurückzuführen ist, dass unter den Büroangestellten mehr Kontaktlinsenträger waren [23] als unter den Bauarbeitern.

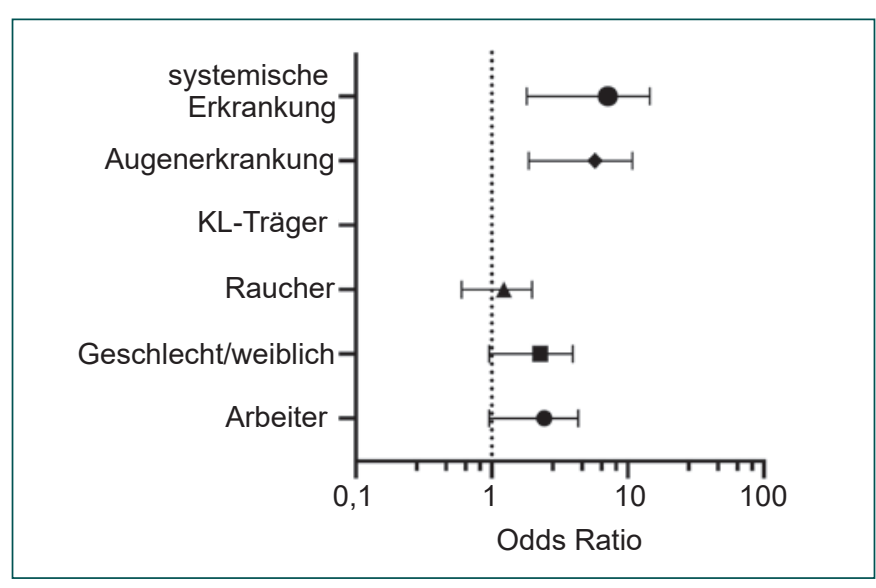

Abb. 2. Odds Ratio der multivariaten DES-Risikoanalyse nach Adjustierung für Arbeitsgruppe, Geschlecht, Rauchgewohnheiten, Kontaktlinsenverwendung sowie okuläre und systemische Erkrankungen.

In der vorliegenden Studie war eine frühere Diagnose einer okulären oder systemischen Erkrankung mit einem erhöhten DESRisiko verbunden. Frühere Studien ergaben eine hohe DES-Prävalenz bei Patienten, die augenärztlich behandelt wurden [25, 26], zusätzlich zu denjenigen mit speziellen Erkrankungen wie Glaukom [8], diabetischer Retinopathie [27] und Erkrankungen der Augenoberfläche [28, 29]. Der Zusammenhang zwischen DES und systemischen Erkrankungen ist umfassend untersucht worden. Paulsen et al. wiesen nach, dass verschiedene Systemerkrankungen wie Allergien, Arthritis, Schilddrüsenerkrankungen und die Einnahme von Medikamenten wie systemisch wirksame Antihistaminika und Steroide mit dem Auftreten eines trockenen Auges in Verbindung stehen [24].

Es liegen Berichte vor, nach denen Rauchen ein Risikofaktor für DES ist [22,30]. Wolkoff zufolge hat Zigarettenrauch Auswirkungen auf den präkornealen Tränenfilm und die Tränenfilmaufreißzeit [23]. Nach Angaben von Lee et al. ist die Prävalenz von Symptomen des trockenen Auges bei Rauchern erhöht [13]. De Kluizenaar et al. [7] und Ranciere et al. [31] wiesen nach, dass Tabakrauch mit einer höheren DES-Prävalenz assoziiert war. In der vorliegenden Studie hatten Raucher niedrigere OSDI-Scores und in der multivariaten Analyse fand sich letztlich kein echter $\mathrm{Zu}$ sammenhang zwischen Rauchen und OSDI-Scores. Die OSDIScores fielen bei Rauchern und Nichtrauchern unterschiedlich aus und die meisten Raucher gehörten zu der Gruppe mit den niedrigeren OSDI-Scores (Bauarbeiter). Im Gegensatz dazu waren die Nichtraucher überwiegend Büroangestellte (die Gruppe mit den höheren OSDI-Scores). Daher nehmen wir an, dass diese Ergebnisse durch die berufliche Exposition der Arbeiter und Angestellten beeinflusst wurden, wie die multivariate Analyse vermuten lässt.

Aufgrund der Art der ausgewählten und untersuchten beruflichen Tätigkeiten war die Studienpopulation bezüglich des Geschlechts heterogen, und dies kann zu Artefakten und Einschränkungen der Studie geführt haben. Diese Studie beinhaltete keine klinische Beurteilung der Augenoberfläche; d.h. es wurden ledig-
Kompass Ophthalmol 2021;7:2-7 DOI: $10.1159 / 000514629$ 
lich die Symptome des trockenen Auges erfasst und keine Erkrankungen der Augenoberfläche oder des trockenen Auges. In Hinblick auf die Ergebnisse, die bei einigen Variablen wie beispielsweise Rauchgewohnheiten und Kontaktlinsenverwendung beobachtet wurden, lässt die begrenzte Stichprobengröße keine sicheren Schlussfolgerungen zu.

Die Ergebnisse dieser Studie unterstreichen, dass es dringend notwendig ist, Menschen, die im Büro arbeiten und den größten Anteil der Erwerbsbevölkerung in den westlichen Ländern ausmachen, über die Risiken, Symptome des trockenen Auges zu entwickeln, zu informieren und Empfehlungen zu geben, wie sich diese minimieren lassen. Zu diesen Maßnahmen sollte gehören, lange ununterbrochene Computerarbeit zu vermeiden, regelmäßige Pausen einzuhalten, um regelmäßiges Blinzeln und die Entspannung der Ziliarmuskeln zu ermöglichen, und eine bessere Kontrolle der Luftfeuchtigkeit am Arbeitsplatz einzufordern.

\section{Schlussfolgerung}

Obwohl Bauarbeiter zahlreichen ungünstigen Arbeitsumgebungsbedingungen ausgesetzt sind, haben sie ein viermal niedrigeres Risiko, Symptome des trockenen Auges zu entwickeln, als Menschen, die in einer durchschnittlichen Büroumgebung arbeiten. Dies unterstreicht die schädlichen Effekte der Büroumgebung auf die Augenoberfläche, die ein erhebliches Risiko für die Entwicklung oder Verschlimmerung von Symptomen des trockenen Auges darstellen.

\section{Beiträge der einzelnen Autoren}

S. H. L. war an der Konzeption dieser Arbeit, an der Gestaltung des Erfassungsbogens und an der Erhebung der Daten durch Befragung der Probanden, an der Erstellung des Artikels, an der Interpretation der Daten bezüglich der bivariaten und multivariaten Analyse sowie an der Überarbeitung der Arbeit zur Einreichung beteiligt. A. K. P. R. war an der Konzeption dieser Arbeit, an der Gestaltung des Erfassungsbogens und an der Erhebung der Daten durch Befragung der Probanden, an der Erstellung des Artikels, an der Interpretation der Daten bezüglich der bivariaten und multivariaten Analyse sowie an der Überarbeitung der Arbeit zur Einreichung beteiligt. P. M. G war an der Erhebung der Daten durch Befragung der Probanden und an der Erstellung des Artikels beteiligt. F. A. war an der Konzeption dieser Arbeit, an der Gestaltung des Erfassungsbogens und an der Analyse und Interpretation aller Daten beteiligt. Außerdem war er an der Erstellung des Artikels sowie an der Überarbeitung der Arbeit zur Einreichung beteiligt. M. G. L. war an der Konzeption dieser Arbeit, an der Gestaltung des Erfassungsbogens, an der Konzeption des Artikels, an der
Analyse und Interpretation der Daten, an der Erstellung der graphischen Informationen beim Schreiben des Artikels, an der Interpretation der Daten bezüglich der bivariaten und multivariaten Analyse sowie an der Überarbeitung der Arbeit zur Einreichung beteiligt. Alle Autoren haben das Manuskript gelesen und freigegeben.

\section{Finanzierung der Studie}

Die Autoren erklären, dass an der Gestaltung und Realisierung dieser Arbeit keine Sponsoren beteiligt waren.

\section{Verfügbarkeit der Daten und Materialien}

Die in dieser Arbeit verwendeten und/oder analysierten Datensätze sind auf begründete Anfrage vom Korrespondenzautor erhältlich.

\section{Genehmigung durch die Ethikkommission und Einwilli- gung zur Teilnahme}

Die Studie wurde durch die Behörden und die Ethikkommission der Universität von Monterrey (UDEM) genehmigt und entsprach den Prinzipien der Deklaration von Helsinki. Nach Aufklärung über die Studie wurde von allen Teilnehmern eine mündliche Einwilligungserklärung eingeholt. Diese Art der Einwilligung wurde von der Ethikkommission der Universität von Monterrey genehmigt, weil viele Studienteilnehmer (insbesondere die Bauarbeiter) nur eingeschränkte Lese- und Schreibfähigkeiten besaßen und weil die Erteilung der informierten Einwilligung am Arbeitsplatz unter suboptimalen Bedingungen und während Arbeitszeit erfolgen musste. Dadurch wurde die Zeit, die für die Einholung der Einwilligung nach Aufklärung und das Interview zur Verfügung stand, erheblich eingeschränkt. Es handelte sich um eine nicht-interventionelle, risikoarme Studie und es wurden keine vertraulichen persönlichen Daten von den Teilnehmern erhoben.

\section{Interessenkonflikte}

Manuel Garza-Leon ist Mitglied des Speaker's Bureau für Alcon. Vortragshonorare von Allergan. Vortragshonorare von SIFI.

Die übrigen Autoren haben keine finanziellen Interessenkonflikte offenzulegen.

\section{Lizenzangabe}

Hernandez-Llamas S, Paz-Ramos AK, Marcos-Gonzalez P, Amparo F, Garza-Leon M: Symptoms of ocular surface disease in construction workers: comparative study with office workers. BMC Ophthalmology 2020; 20:272. (DOI: $10.1186 / \mathrm{s} 12886-020-01548-0)$. ${ }^{\circ} 2020$ The Author(s). (Übersetzung; Abbreviations, Consent for publication und Publisher's Note gekürzt), lizensiert unter CC BY 4.0 (https://creativecommons.org/licenses/by/4.0/deed.de).

\section{Literatur}

Die Literatur ist unter www.karger.com/doi/10.1159/000514629 abrufbar. 